\title{
Six-month color change and water sorption of 9 new-generation flowable composites in 6 staining solutions
}

\section{Maria ARREGUI (a) \\ Luis GINER(a) \\ Marco FERRARI (b) \\ Marta VALLÉS(a) \\ Montserrat MERCADÉ(a)}

(a) Universitat Internacional de Catalunya, Department of Restorative Dentistry and Endodontics, Barcelona, Spain.

(b) University of Siena, Policlinico Le Scotte, Department of Medical Biotechnologies, Siena, Italia.

Declaration of Interests: The authors certify that they have no commercial or associative interest that represents a conflict of interest in connection with the manuscript.

Corresponding Author:

Montserrat Mercadé

E-mail:mmercade@uic.es

DOI: 10.1590/1807-3107BOR-2016.vol30.0123

Submitted: Jan 27, 2016

Accepted for publication: Aug 29, 2016

Last revision: Sep 15, 2016
Abstract: Color match and water sorption are two factors that affect restorative materials. Discoloration is essential in the lifespan of restorations. The aim of this study was to evaluate color change and water sorption of nine flowable composites at multiple time points over 6 months. 60 samples of each composite were divided into two groups (Color Change and Water Sorption/Solubility). Each Color Change group was divided into six subgroups, which were immersed in distilled water (DW), coffee (CF), Coca-Cola (CC), red wine (RW), tea (TE) and orange juice (OJ). The color was measured at the baseline, $1,2,3$ and 4 weeks, and 3 and 6 months and color change values $(\Delta \mathrm{E})$ were calculated. Each Water Sorption $\left[\mathrm{W}_{\mathrm{S}}\right] /$ Solubility $\left[\mathrm{W}_{\mathrm{L}}\right]$ group was tested according to ISO 4049:2009. The data were evaluated using two-way ANOVA, Fisher's post-hoc test and Pearson's correlation test. The composite with the lowest $\Delta \mathrm{E}$ differed for each solution: Filtek ${ }^{\mathrm{TM}}$ Bulk Fill in DW $(\Delta \mathrm{E}=0.73(0.17-1.759))$; Vertise Flow in $\mathrm{CF}(\Delta \mathrm{E}=14.75$ (7.91-27.41)), in TE $(\Delta \mathrm{E}=7.27(2.81-24.81))$ and $\mathrm{OJ}(\Delta \mathrm{E}=3.17(0.87-9.92))$; Tetric EvoFlow ${ }^{\circledR}$ in $\mathrm{CC}(\Delta \mathrm{E}=1.27(0.45-4.02))$; and Filtek ${ }^{\mathrm{TM}}$ Supreme XTE in RW ( $\Delta \mathrm{E}=8.88$ (5.23-19.59)). RW caused the most discoloration $(\Delta \mathrm{E}=23.62$ (4.93-51.36)). Vertise Flow showed the highest water sorption $\left(\mathrm{W}_{\mathrm{S}}=69.10 \pm 7.19\right)$. The Pearson test showed statistically significant positive correlations between water sorption and solubility and between water sorption and $\Delta \mathrm{E}$; the positive solubility- $\Delta \mathrm{E}$ correlation was not statistically significant. The findings suggest that water sorption is one factor associated with the ability of composites to discolor; however, discoloration is a multifactorial problem.

Keywords: Flowable Hybrid Composite; Dental Materials.

\section{Introduction}

Composite resin-based materials are widely used to meet the growing demand for aesthetic and cosmetic dental treatments. ${ }^{1,2}$ These composites have a natural appearance and are more conservative and less costly than other materials, such as ceramics. ${ }^{3,4}$ Several types of resin-based composites are available with different physical properties and are classified according to resin matrix, ${ }^{5}$ particle size, filler distribution ${ }^{6}$ and flow ability. ${ }^{6,7}$

The first generation of flowable composites was introduced in 1996. ${ }^{7}$ These low-viscosity resin-based restorative materials differ from conventional resin composites in their filler load ${ }^{8}$ and in their 
formulation, which contains a higher proportion of diluent monomers. ${ }^{8}$ The novel flowable composites were developed in 2000 with the aim of improving their mechanical properties. ${ }^{9}$ These flowable composites are available in two forms: self-adhesive and bulk-fill flowable composites. Self-adhesive flowable composites contain acidic monomers ${ }^{10,11}$ and require no adhesive bonding agent, necessitating fewer clinical application steps than conventional composites. ${ }^{12}$ Bulk-fill flowable composites can be placed in bulk up to $4 \mathrm{~mm}$ thick, eliminating the incremental placement technique required with other currently available composites. ${ }^{13}$

The differences in composition and filler content are key to the optical properties of resin composites: flowable composites exhibit different optical and color properties than conventional composites..$^{14}$ These differences, which are related to minor pigment additions ${ }^{15}$ and their major levels of translucency, ${ }^{14}$ have a greater effect on the color change of flowable composites than on universal composites. ${ }^{14}$ One of the main drawbacks of resin composites is their tendency to change color due to intrinsic and extrinsic factors ${ }^{1,2,4,16,17,18}$ after long periods in the oral environment and this discoloration could make the color of the resin composites perceptible to the human eye, as they would no longer match the color of the tooth. ${ }^{4,16,17}$ Based on the human eye's ability to perceive color differences, three intervals were used to distinguish changes in color: $\Delta \mathrm{E}<1$ (imperceptible to the human eye), ${ }^{2} \Delta \mathrm{E}$ from 1-3.3 (visible only to the skilled observer, clinically acceptable), ${ }^{1,2,3}$ and $\Delta \mathrm{E}>3.3$ (easy to discern, not clinically acceptable)., ${ }^{1,3,19}$

Numerous in vitro studies have demonstrated that common drinks such as coffee,,$^{19,20,21,22,23}$ tea, ${ }^{2,3,18,23,24}$ red wine, ${ }^{1,3,4,24}$ orange juice, ${ }^{1,3,24}$ and cola drinks ${ }^{1,2,3,4}$ can cause significant discoloration of composite resin materials.

In a wet oral environment, composites may absorb water or other substances such as saliva, food components or beverages, which can have an important influence on the degradation of dental composites. ${ }^{25}$ Since water sorption is a diffusion-controlled, time-dependent process, ${ }^{11}$ it may decrease the lifespan of the restoration by expanding and plasticizing the resin component and hydrolyzing the silane. ${ }^{4,25}$ Water sorption is associated with solubility, which consists of the release of residual products such as monomers and oligomers. ${ }^{25}$ These leached products alter the microstructure of the matrix, creating voids and microcrack formation $s^{10}$ that allow stain penetration and discoloration. ${ }^{2,4}$

In response to the lack of research on the color instability of conventional flowable composites ${ }^{14,15}$ and the apparent absence of literature on new flowable composites, this study aimed to evaluate the effects of immersion in a range of beverages on the color change and water sorption of nine flowable composites at multiple time points over 6 months. The first null hypothesis was that increased color change is not related to water sorption in composites maintained at $37^{\circ} \mathrm{C}$ for 6 months. The second null hypothesis was that increased color change is related to staining solutions in composites maintained at $37^{\circ} \mathrm{C}$ for 6 months.

\section{Methodology}

\section{Disk specimen preparation}

The nine flowable composites tested in this study are described in Table 1. The shades were A2 and Universal (U). Sixty specimens for each composite were produced using two different silicone molds (Contrast, VOCO, Cuxhaven, Germany; LOT: 1118534): first, color change specimens $(\mathrm{n}=30) 10 \pm 1 \mathrm{~mm}$ in diameter $\times 2 \pm 0.1 \mathrm{~mm}$ thick, yielding the Color Change group; and second, water sorption specimens $(\mathrm{n}=30) 15 \pm 1 \mathrm{~mm}$ in diameter $\times 1 \pm 0.1 \mathrm{~mm}$ thick, yielding the Water Sorption/Solubility group. The impression material was not known to contain any leachable plasticizer or other material that would potentially contaminate the surfaces of the specimens.

After filling each mold with the material and covering it with a sheet of polyester film, the top of the material was compressed using a glass plate. The specimens were light-cured on both sides for 60 s (Demi; SDS Kerr Corp., Middleton WI, USA; $1167 \mathrm{~mW} / \mathrm{cm}^{2}$ ). A calibrated radiometer (Bluephase; Ivoclar Vivadent AG, Schaan, Liechtenstein) was 
Table 1. Materials tested.

\begin{tabular}{|c|c|c|c|c|c|c|}
\hline Code & Brand Name & Composite type & Compositions & Shade & Batch \#LOT & Manufacturer \\
\hline PF & Premise flowable & $\begin{array}{l}\text { Nanohybrid } \\
\text { flow }\end{array}$ & $\begin{array}{c}\text { Matrix: } 9 \\
\text { EBPDMA, TEGDMA } \\
\text { Filler (84wt\%, 70vol\%): } \\
\text { Prepolymerized filler (PPF), bBarium glass, } \\
\text { silica filler }\end{array}$ & $\mathrm{A} 2$ & LOT 476526 & $\begin{array}{l}\text { Kerr Hawe } \\
\text { S.A, Bioggio, } \\
\text { Switzerland }\end{array}$ \\
\hline VF & Vertise flow & $\begin{array}{l}\text { Self-adhesive } \\
\text { flow }\end{array}$ & $\begin{array}{l}\text { Matrix: } ¥ \\
\text { GPDM and methacrylate co-monomers } \\
\text { Filler (70wt\%, 48vol\%): } \\
\text { Prepolymerized filler, barium glass, nano- } \\
\text { sized colloidal silca, nano-sized ytterbium } \\
\text { fluoride }\end{array}$ & $\mathrm{A} 2$ & LOT 4732395 & $\begin{array}{l}\text { Kerr Hawe } \\
\text { S.A, Bioggio, } \\
\text { Switzerland }\end{array}$ \\
\hline SF & Sonic fill & Bulk fill & $\begin{array}{c}\text { Matrix: } \dagger \\
\text { Bis-GMA, TEGDMA, Bis-EMA, SIMA } \\
\text { Filler (84w+\%, 66.8vol\%) }\end{array}$ & $\mathrm{A} 2$ & LOT 4735797 & $\begin{array}{l}\text { Kerr Hawe } \\
\text { S.A, Bioggio, } \\
\text { Switzerland }\end{array}$ \\
\hline VBF & Venus bulk fill & Bulk fill & $\begin{array}{c}\text { Matrix:Ş } \\
\text { UDMA, EBPDMA } \\
\text { Filler (65w+\%, 38vol\%): } \\
\text { Ba-Al-F-Si Glass, SiO2 }\end{array}$ & U & LOT 010101 & $\begin{array}{l}\text { Heraeus Kulzer, } \\
\text { Hanau, Germany }\end{array}$ \\
\hline VDF & $\begin{array}{c}\text { Venus diamond } \\
\text { flow }\end{array}$ & $\begin{array}{l}\text { Nanohybrid } \\
\text { flow }\end{array}$ & $\begin{array}{c}\text { Matrix: } \beta \\
\text { UDMA, EBADMA } \\
\text { Filler }(65 w+\%, 4 \text { lvol\%): } \\
\text { Ba-Al-F-silicate glass, YbF3, SiO2 }\end{array}$ & A2 & LOT 010105 & $\begin{array}{l}\text { Heraeus Kulzer, } \\
\text { Hanau, Germany }\end{array}$ \\
\hline TEF & Tetric evoflow & $\begin{array}{l}\text { Nanohybrid } \\
\text { flow }\end{array}$ & $\begin{array}{c}\text { Matrix: } \neq \\
\text { Bis-GMA, UDMA, TEGDMA } \\
\text { Filler (58wt\%, 30.7vol\%): } \\
\text { Barium glass, YbF3, Ba-Al-Fluorosilicate } \\
\text { glass, } \mathrm{SiO} 2\end{array}$ & $\mathrm{~A} 2$ & LOT S13466 & $\begin{array}{l}\text { Ivoclar Vivadent, } \\
\text { Schaan, } \\
\text { Liechtenstein }\end{array}$ \\
\hline SDR & SDR & Bulk fill & $\begin{array}{c}\text { Matrix:\# } \\
\text { UDMA, TEGDMA, EBPDMA } \\
\text { Filler (68w+\%, 44vol\%): } \\
\text { Ba-Al-F-B-Si glass, St-Al-F-Si glass }\end{array}$ & $U$ & $\begin{array}{c}\text { LOT } \\
1301000164\end{array}$ & $\begin{array}{c}\text { Dentsply, Konstanz, } \\
\text { Germany }\end{array}$ \\
\hline FSX & Filtek supreme XTE & $\begin{array}{l}\text { Nanohybrid } \\
\text { flow }\end{array}$ & $\begin{array}{c}\text { Matrix:* } \\
\text { Bis-GMA, TEGDMA, Bis-EMA } \\
\text { Filler (58-82wt\%, 46vol\%): } \\
\text { Ceramic (52-60wt\%) } \\
\text { SiO2 (3-1 1wt\%) } \\
\text { ZrOx (3-1 1wt\%) }\end{array}$ & A2 & LOT N432599 & $\begin{array}{c}\text { 3M ESPE, St. Paul, } \\
\text { MN, USA }\end{array}$ \\
\hline FBF & Filtek bulk fill & Bulk fill & $\begin{array}{c}\text { Matrix: } \S \\
\text { Bis-GMA, Bis-EMA, UDMA, Procrylat resins. } \\
\text { Filler (64.5wt\%, 42.5vol\%): } \\
\text { Ytterbium trifluoride filler, zirconia/silica }\end{array}$ & $U$ & LOT N426221 & $\begin{array}{c}\text { 3M ESPE, St. PAUI, } \\
\text { MN, USA }\end{array}$ \\
\hline
\end{tabular}

EBPDMA: ethoxylated bisphenol A dimethacrylate; TEGDMA: triethylene glycol dimethacrylate; GPDM: glycerol phosphate dimethacrylate; Bis-GMA: bisphenol A diglycidy ether dimethacrylate; Bis-EMA: bisphenol A polyethylene glycol diether dimethacrylate; UDMA: urethane dimethacrylate. II Kerr, Directions for use, http://www.kerrdental.com/cms-filesystem-action/KerrDental-Products-DFU/ dfu-premiseflowable-78725.pdf;

$¥$ 2010, Kerr Technical Bulletin, http://www.kerrdental.com/cms-filesystem-action/KerrDental-products-techinfo/vertiseflow_techbulletin_34929b.pdf; † Kerr, Directions for use, http://www.kerrdental.com/cms-filesystem-action?file=/kerrdental-products-dfu/2011 SonicFill_\%5BNA\%5D.pdf; \$̧ 2010, Heraeus Kuzer, Instructions for use, http://webmedia.kulzer-dental.com/media/hkg/downloads_new/venus_5/venus_bulk_fill_1/GBA_ Venus_Bulk_Fill_INT.pdf; $\beta$ 2009, Heraeus Kulzer, Instructions for use, http://webmedia.kulzer-dental.com/media/hkg/downloads_new/venus_5/venus_diamond_flow_2/ GBA_Venus_Diamond_flow_INT.pdf;

$\ddagger 20 \overline{1} 1$, Ivoclar Vivadent, Instructions for use, http://www.ivoclarvivadent.us/en-us/products/restorative-materials/composites/tetric-evoflow; \# 2014, Dentsply, Instructions for use, https://www.dentsply.co.uk/Products/Instructions-for-use.aspx

* 2014, 3M ESPE, Instructions for use, http://multimedia.3m.com/mws/media/573715O/filtek-supreme-xte-instructions-for-use-western-europe.pdf; $\S 2014,3$ M ESPE, Technical Bochure, http://solutions.3mae.ae/wps/portal/3M/en_AE/3M_ESPE/Dental-Manufacturers/Products/DentalRestorative-Materials/Dental-Composites/Flowable-Restorative/\#tab5 
used to verify the intensity of the light-curing unit. The periphery of all specimens was polished with 1200-grit silicon carbide paper to remove flash and irregularities and the surfaces were left unpolished following the ISO 4049:2009 recommendation. ${ }^{26}$ The specimens were stored in the dark at room temperature until testing.

\section{Color change}

\section{Immersion of samples in solution}

To evaluate the color change, the Color Change group $(\mathrm{n}=30)$ for each composite was divided into six subgroups of five specimens, which were immersed in six different solutions: (distilled water (DW) $(\mathrm{pH}=5.84 \pm 0.45$; Quicesa, Spain), coffee (CF) ( $\mathrm{pH}=4.58 \pm 0.09$; Nescafé Classic, Nestlé SA, Vevey, Switzerland), Coca-Cola (CC) ( $\mathrm{pH}=2.50 \pm 0.17$; The Coca-Cola Company, Atlanta, GA, USA), red wine $(\mathrm{RW})(\mathrm{pH}=3.55 \pm 0.14$; Cariñena, Spain), tea (TE) ( $\mathrm{pH}=4.69$; English Breakfast, Twinings ${ }^{\mathrm{TM}}$, London, England), and orange juice (OJ) $(\mathrm{pH}=3.28 \pm 0.17$; Don Simón, Spain). The distilled water served as a control. To insure that all surfaces were fully exposed to the test solutions, the specimens of each composite were individually immersed in vials containing $2 \mathrm{~mL}$ of each solution and stored at $37^{\circ} \mathrm{C}$. The solutions were renewed every 2 days and $\mathrm{pH}$ measurements were recorded ( $\mathrm{pH}$ Meter Basic 20; Crison Instruments SA, Alella, Spain) before re-immersing the specimens.

\section{Assessment of color change}

The baseline color measurements were recorded for all specimens according to the Commission International de l'Eclairage (CIE) L*a* ${ }^{*}$ color scale relative to the CIE standard illuminant D65 over a black background, using a reflection spectrophotometer (SpectroShade MHT; MHT S.p.A., Arbizzano di Negrar, Italy). The CIE L*a* $b^{*}$ color system is a three-dimensional color measurement method, where $L^{*}$ refers to the lightness coordinate, which ranges in value from 0 (black)-100 (white), and $a^{*}$ and $b^{*}$ are chromaticity coordinates on the green-red $\left(-\mathrm{a}^{*}=\right.$ green; $\left.+\mathrm{a}^{*}=\mathrm{red}\right)$ and blue-yellow $\left(-\mathrm{b}^{*}=\right.$ blue; $+b^{*}=$ yellow) axes, respectively. ${ }^{1,4}$
All specimens were rinsed with distilled water and blotted dry with absorbent paper before measurement. Three measurements were obtained from each specimen by a single operator and were recorded at baseline (T0), 1 week (T1), 2 weeks (T2), 3 weeks (T3) and 4 weeks (T4), 3 months (T5), and 6 months (T6). Before each color measurement, the spectrophotometer was calibrated according to the manufacturer's instructions. The overall color change values $(\Delta \mathrm{E})$ were calculated as follows:

Equation 1: $\mathrm{CIEL}^{*} \mathrm{a}^{*} \mathrm{~b}^{*} \Delta \mathrm{E}_{\mathrm{a}, \mathrm{b}}=\left[\left(\Delta \mathrm{L}^{*}\right)^{2}+\left(\Delta \mathrm{a}^{*}\right)^{2}+\left(\Delta \mathrm{b}^{*}\right)^{2}\right]^{1 / 2}$

where $\Delta \mathrm{L}^{*}$ is lightness and $\Delta \mathrm{a}^{*}$ and $\Delta \mathrm{b}^{*}$ are the differences in the green-red and blue-yellow axes, respectively. The staining was considered clinically unacceptable when $\Delta \mathrm{E}$ values were $\geq 3.3$., 24

\section{Water sorption/solubility}

The water sorption/solubility testing was performed on the Water Sorption/Solubility group $(n=30)$ for each composite according to ISO 4049:2009. ${ }^{26}$ Each specimen was weighed, transferred to a desiccator containing dehydrated silica gel (Químics Dalmau SL, Barcelona, Spain) and maintained at $37 \pm 1^{\circ} \mathrm{C}$ for $22 \mathrm{~h}$ then $23 \pm 1^{\circ} \mathrm{C}$ for $2 \mathrm{~h}$. The specimens were reweighed and the conditioning cycle was repeated until the decrease in mass of each specimen $\left(m_{1}\right)$ was constant. After the conditioning cycle, the diameter and thickness of each specimen was measured three times with a caliper to calculate the specimen volume (V) in $\mathrm{mm}^{3}$. To ensure complete exposure, each specimen was suspended in an individual vial containing $20 \mathrm{~mL}$ of distilled water and maintained at $37 \pm 1^{\circ} \mathrm{C}$ for the same period as that described for color measurement.

Following the immersion period, the specimens were removed from the vial, excess water was removed with absorbent paper, and the specimen was reweighed $\left(\mathrm{m}_{2}\right)$. The immersed specimens were then subjected to the aforementioned conditioning cycle until the decrease in the mass of each specimen $\left(\mathrm{m}_{3}\right)$ was constant.

The water sorption $\left(\mathrm{W}_{\mathrm{S}}\right)$ and water solubility $\left(\mathrm{W}_{\mathrm{L}}\right)$ of each specimen were calculated in accordance with ISO4049:2009 ${ }^{26}$ using the following formula:

Equation 2: Water sorption $\mathrm{W}_{\mathrm{S}}=\left(\mathrm{m}_{2}-\mathrm{m}_{3}\right) / \mathrm{V}$

Equation 3: Water solubility $\mathrm{W}_{\mathrm{L}}=\left(\mathrm{m}_{1}-\mathrm{m}_{3}\right) / \mathrm{V}$ 
ISO4049:200926 was considered acceptable when $\mathrm{W}_{\mathrm{S}}$ was $\leq 40 \mu \mathrm{g} / \mathrm{mm}^{3}$ and $\mathrm{W}_{\mathrm{L}}$ was $\leq 7.5 \mu \mathrm{g} / \mathrm{mm}^{3}$.

\section{Statistical analysis}

The $\Delta \mathrm{E}$ observed for the different resin composites (all measurements were performed in triplicate), beverages and times were subjected to 2-way repeated measures analysis of variance (ANOVA) and Fisher' post-hoc test was used for multiple comparisons between groups. These two tests were also used to analyze the water sorption and solubility of resin composites kept in distilled water for 6 months. All tests were performed with a significance level of 95\% using StatGraphics Centurion XV (StatPoint Technologies, Inc., Warrenton, VA, USA). Pearson's correlation test was performed to determine possible correlations between water sorption and solubility, water sorption and color change, and solubility and color change.

\section{Results}

\section{Color change}

The 2-way repeated measures ANOVA was statistically significant ( $\mathrm{p}$-value $<0.001$ ) in the following interactions: composite-staining solution; composite-time, and time-staining solution. The data for $\Delta \mathrm{E}$ were non-parametric and were calculated as the median (Minimum-Maximum); however, a logarithmic transformation of the $\Delta \mathrm{E}$ values was conducted, the Kolmogorov-Smirnov test showed normality of the transformed data and the ANOVA test maintained the same levels of significance.

Table 2 summarizes the median (Minimum and Maximum) of the $\Delta \mathrm{E}$ for the nine composites after immersion in the solutions and Figure shows the color change progression of each composite and staining solution at the different time points. The composite with the lowest $\Delta \mathrm{E}$ value differed for each solution. In distilled water, Filtek ${ }^{\mathrm{TM}}$ Bulk Fill (FBF; 3M ESPE; St. Paul, MN, USA) exhibited the lowest $\Delta \mathrm{E}$ for all time points; in coffee, Vertise Flow (VF; Kerr Hawe S. A., Bioggio, Switzerland) exhibited the lowest $\Delta \mathrm{E}$ for $\mathrm{T} 1$ and $\mathrm{T} 4$ and Filtek ${ }^{\mathrm{TM}}$ Supreme XTE Flow (FSXT; 3M ESPE) for T2, T3, T5 and T6; in Coca-Cola, Tetric EvoFlow ${ }^{\circledR}$ (TEF; Ivoclar
Vivadent AG, Schaan, Liechtenstein) exhibited the lowest $\Delta \mathrm{E}$ for T1-T4 and Venus Diamond Flow (Heraeus Kulzer, Hanau, Germany) for T5 and T6; in tea, Vertise Flow (VF; Kerr Hawe S. A., Bioggio, Switzerland) exhibited the lowest $\Delta \mathrm{E}$ for T1-T4 and Filtek $^{\text {TM }}$ Supreme XTE Flow (FSXT; 3M ESPE) for T5 and T6; in red wine, Premise Flowable (Kerr Hawe, S.A., Bioggio, Switzerland) for T1 and Filtek $^{\mathrm{TM}}$ Supreme XTE Flow (FSXT; 3M ESPE) for T2-T6; and in orange juice, VF exhibited the lowest $\Delta \mathrm{E}$ for all time points.

The Fisher's post-hoc test revealed differences between all staining solutions. The lowest values were obtained for distilled water $(\Delta \mathrm{E}=1.85(0.17-9.03))$ and Coca-Cola $(\Delta \mathrm{E}=2.28(0.33-16.52))$, intermediate values were obtained for orange juice $(\Delta \mathrm{E}=6.76$ (0.87-24.35)), and the highest values were obtained for tea $(\Delta \mathrm{E}=20.36(2.81-63.99))$, coffee $(\Delta \mathrm{E}=20.77$ (7.43-44.12)), and red wine $(\Delta \mathrm{E}=23.62(4.93-51.36))$.

Significant differences were evident when the materials were compared according to composite type. The lowest $\Delta \mathrm{E}$ was obtained for nanohybrid materials $(\Delta \mathrm{E}=8.55(0.22-63.39))$, an intermediate $\Delta \mathrm{E}$ was obtained for self-adhesive composites $(\Delta \mathrm{E}=9.35$ (0.51-51.36)), and the highest $\Delta \mathrm{E}$ was obtained for bulk-fill composites $(\Delta \mathrm{E}=14.21(0.17-51.09))$.

\section{Water sorption and solubility}

Table 3 shows the water sorption, solubility $\left(\mu \mathrm{g} / \mathrm{mm}^{3}\right)$ and results of statistical analysis after 6 months' immersion in distilled water at $37^{\circ} \mathrm{C}$. The results for water sorption, from lowest to highest, were SonicFill ${ }^{\mathrm{TM}}$ (SF; Kerr Corp.) < Smart Dentine Replacement ${ }^{\circledR}$ (SDR; Denstply DeTrey GmbH, Konstanz, Germany) < Venus ${ }^{\circledR}$ Bulk Fill (VBF; Heraeus Kulzer GmbH, Hanau, Germany) = Venus ${ }^{\circledR}$ Diamond Flow (VDF; Heraeus Kulzer $\mathrm{GmbH}$ ) $=$ FBF $=$ FSXT $<$ Premise Flowable (PF; Kerr Corp.) < TEF < VF. The results for solubility, from lowest to highest, were $\mathrm{VBF}<\mathrm{FBF}=\mathrm{PF}<\mathrm{VDF}<\mathrm{FSXT}<\mathrm{SDR}<\mathrm{TEF}=\mathrm{SF}<\mathrm{VF}$. The lowest water sorption values were obtained with SF at all time points evaluated. Only VF showed values of water sorption and solubility higher than those stipulated by ISO 4049:2009. ${ }^{26}$ 
Six-month color change and water sorption of 9 new-generation flowable composites in 6 staining solutions

Table 2. Results of $\Delta \mathrm{E}$ - Median (Minimun- Maximum).

\begin{tabular}{|c|c|c|c|c|c|c|c|}
\hline Staining solution & Composite & $\mathrm{T1}$ & T2 & T3 & $\mathrm{T} 4$ & T5 & T6 \\
\hline \multirow{9}{*}{ Distilled water } & $\mathrm{PF}$ & $\begin{array}{c}1.05 \\
(0.43-4.26)\end{array}$ & $\begin{array}{c}1.40 \\
(0.74-5.36)\end{array}$ & $\begin{array}{c}1.41 \\
(0.88-4.58)\end{array}$ & $\begin{array}{c}1.44 \\
(0.96-4.75)\end{array}$ & $\begin{array}{c}1.94 \\
(1.61-3.16)\end{array}$ & $\begin{array}{c}2.18 \\
(1.61-3.91)\end{array}$ \\
\hline & SDR & $\begin{array}{c}1.14 \\
(0.22-3.27)\end{array}$ & $\begin{array}{c}1.40 \\
(0.31-2.81)\end{array}$ & $\begin{array}{c}2.14 \\
(0.76-3.29)\end{array}$ & $\begin{array}{c}2.35 \\
(0.44-3.79)\end{array}$ & $\begin{array}{c}2.12 \\
(0.82-4.08)\end{array}$ & $\begin{array}{c}1.98 \\
(1.40-2.85)\end{array}$ \\
\hline & VBF & $\begin{array}{c}4.76 \\
(3.52-8.37)^{*}\end{array}$ & $\begin{array}{c}3.49 \\
(2.80-4.80)^{*}\end{array}$ & $\begin{array}{c}3.54 \\
(2.70-9.03)^{*}\end{array}$ & $\begin{array}{c}3.00 \\
(2.67-4.74)\end{array}$ & $\begin{array}{c}3.98 \\
(2.82-7.74)^{*}\end{array}$ & $\begin{array}{c}4.94 \\
(3.29-6.01)^{*}\end{array}$ \\
\hline & FSXT & $\begin{array}{c}1.06 \\
(0.35-1.54)\end{array}$ & $\begin{array}{c}0.91 \\
(0.42-1.46)\end{array}$ & $\begin{array}{c}0.86 \\
(0.22-2.12)\end{array}$ & $\begin{array}{c}1.12 \\
(0.41-1.99)\end{array}$ & $\begin{array}{c}1.50 \\
(0.85-2.93)\end{array}$ & $\begin{array}{c}1.60 \\
(1.03-2.36)\end{array}$ \\
\hline & VDF & $\begin{array}{c}2.37 \\
(1.93-3.04)\end{array}$ & $\begin{array}{c}2.49 \\
(2.01-2.98)\end{array}$ & $\begin{array}{c}2.44 \\
(1.76-3.57)\end{array}$ & $\begin{array}{c}2.45 \\
(1.72-3.21)\end{array}$ & $\begin{array}{c}2.26 \\
(1.49-3.72)\end{array}$ & $\begin{array}{c}2.50 \\
(1.50-3.90)\end{array}$ \\
\hline & VF & $\begin{array}{c}1.85 \\
(1.08-3.47)\end{array}$ & $\begin{array}{c}2.32 \\
(1.24-2.82)\end{array}$ & $\begin{array}{c}2.30 \\
(1.25-2.75)\end{array}$ & $\begin{array}{c}2.29 \\
(1.27-3.46)\end{array}$ & $\begin{array}{c}2.35 \\
(1.16-3.83)\end{array}$ & $\begin{array}{c}2.76 \\
(1.96-4.69)\end{array}$ \\
\hline & FBF & $\begin{array}{c}0.61 \\
(0.27-1.69)\end{array}$ & $\begin{array}{c}0.66 \\
(0.30-1.75)\end{array}$ & $\begin{array}{c}0.75 \\
(0.17-1.74)\end{array}$ & $\begin{array}{c}0.72 \\
(0.32-1.26)\end{array}$ & $\begin{array}{c}0.95 \\
(0.31-1.49)\end{array}$ & $\begin{array}{c}0.78 \\
(0.38-1.21)\end{array}$ \\
\hline & TEF & $\begin{array}{c}1.22 \\
(0.47-2.58)\end{array}$ & $\begin{array}{c}1.47 \\
(0.48-2.24)\end{array}$ & $\begin{array}{c}1.48 \\
(0.59-2.27)\end{array}$ & $\begin{array}{c}1.47 \\
(0.95-2.43)\end{array}$ & $\begin{array}{c}1.75 \\
(1.05-2.88)\end{array}$ & $\begin{array}{c}1.85 \\
(1.47-2.68)\end{array}$ \\
\hline & SF & $\begin{array}{c}1.19 \\
(0.30-2.09)\end{array}$ & $\begin{array}{c}2.00 \\
(0.93-2.82)\end{array}$ & $\begin{array}{c}1.89 \\
(1.32-2.75)\end{array}$ & $\begin{array}{c}1.92 \\
(1.09-2.93)\end{array}$ & $\begin{array}{c}1.92 \\
(0.81-3.47)\end{array}$ & $\begin{array}{c}1.81 \\
(1.30-2.94)\end{array}$ \\
\hline \multirow{9}{*}{ Coffee } & PF & $\begin{array}{c}10.59 \\
(9.25-13.93)^{*}\end{array}$ & $\begin{array}{c}14.00 \\
(12.93-16.78)^{*}\end{array}$ & $\begin{array}{c}16.16 \\
(14.65-18.73)^{*}\end{array}$ & $\begin{array}{c}17.43 \\
(15.51-18.94)^{*}\end{array}$ & $\begin{array}{c}22.97 \\
(18.66-27.71)^{*}\end{array}$ & $\begin{array}{c}22.93 \\
(19.50-29.98)^{*}\end{array}$ \\
\hline & SDR & $\begin{array}{c}19.09 \\
(15.64-21.61)^{*}\end{array}$ & $\begin{array}{c}23.91 \\
(20.34-28.27)^{*}\end{array}$ & $\begin{array}{c}25.96 \\
(22.27-28.80)^{*}\end{array}$ & $\begin{array}{c}27.55 \\
(24.44-31.13)^{*}\end{array}$ & $\begin{array}{c}34.34 \\
(31.45-37.89)^{*}\end{array}$ & $\begin{array}{c}34.80 \\
(29.69-38.93)^{*}\end{array}$ \\
\hline & VBF & $\begin{array}{c}24.86 \\
(22.34-27.84)^{*}\end{array}$ & $\begin{array}{c}30.85 \\
(27.60-34.27)^{*}\end{array}$ & $\begin{array}{c}33.64 \\
(30.34-36.67)^{*}\end{array}$ & $\begin{array}{c}35.60 \\
(32.28-38.37)^{*}\end{array}$ & $\begin{array}{c}41.59 \\
(38.43-44.12)^{*}\end{array}$ & $\begin{array}{c}41.79 \\
(38.65-43.94)^{*}\end{array}$ \\
\hline & FSXT & $\begin{array}{c}8.93 \\
(7.43-10.41)^{*}\end{array}$ & $\begin{array}{c}11.54 \\
(9.23-14.49)^{*}\end{array}$ & $\begin{array}{c}14.29 \\
(12.84-17.99)^{*}\end{array}$ & $\begin{array}{c}15.43 \\
(14.41-17.98)^{*}\end{array}$ & $\begin{array}{c}19.10 \\
(17.56-23.04)^{*}\end{array}$ & $\begin{array}{c}19.30 \\
(15.78-24.10)^{*}\end{array}$ \\
\hline & VDF & $\begin{array}{c}20.35 \\
(18.42-22.57)^{*}\end{array}$ & $\begin{array}{c}24.79 \\
(23.70-26.64)^{*}\end{array}$ & $\begin{array}{c}28.37 \\
(27.49-30.24)^{*}\end{array}$ & $\begin{array}{c}30.59 \\
(29.77-32.47)^{*}\end{array}$ & $\begin{array}{c}37.75 \\
(34.95-40.12)^{*}\end{array}$ & $\begin{array}{c}37.96 \\
(37.02-40.20)^{*}\end{array}$ \\
\hline & VF & $\begin{array}{c}8.77 \\
(7.91-10.80)^{*}\end{array}$ & $\begin{array}{c}12.00 \\
(10.74-13.72)^{*}\end{array}$ & $\begin{array}{c}14.43 \\
(12.93-15.62)^{*}\end{array}$ & $\begin{array}{c}15.38 \\
(13.98-16.86)^{*}\end{array}$ & $\begin{array}{c}20.36 \\
(18.51-21.79)^{*}\end{array}$ & $\begin{array}{c}25.63 \\
(24.25-27.41)^{*}\end{array}$ \\
\hline & FBF & $\begin{array}{c}15.47 \\
(13.70-16.65)^{*}\end{array}$ & $\begin{array}{c}18.96 \\
(17.45-20.28)^{*}\end{array}$ & $\begin{array}{c}21.66 \\
(20.10-21.13)^{*}\end{array}$ & $\begin{array}{c}23.22 \\
(21.25-24.60)^{*}\end{array}$ & $\begin{array}{c}27.69 \\
(25.57-29.43)^{*}\end{array}$ & $\begin{array}{c}30.04 \\
(28.09-30.97)^{*}\end{array}$ \\
\hline & TEF & $\begin{array}{c}11.00 \\
(9.30-13.41)^{*}\end{array}$ & $\begin{array}{c}13.55 \\
(11.54-14.99)^{*}\end{array}$ & $\begin{array}{c}15.35 \\
(13.64-17.99)^{*}\end{array}$ & $\begin{array}{c}16.20 \\
(14.62-18.60)^{*}\end{array}$ & $\begin{array}{c}20.20 \\
(18.54-22.64)^{*}\end{array}$ & $\begin{array}{c}24.79 \\
(22.33-27.78)^{*}\end{array}$ \\
\hline & SF & $\begin{array}{c}12.04 \\
(10.71-13.80)^{*}\end{array}$ & $\begin{array}{c}14.16 \\
(13.01-16.47)^{*}\end{array}$ & $\begin{array}{c}16.31 \\
(14.24-18.04)^{*}\end{array}$ & $\begin{array}{c}17.12 \\
(15.97-18.77)^{*}\end{array}$ & $\begin{array}{c}22.18 \\
(20.40-25.13)^{*}\end{array}$ & $\begin{array}{c}25.01 \\
(22.81-27.31)^{*}\end{array}$ \\
\hline \multirow{9}{*}{ Coca-Cola } & PF & $\begin{array}{c}1.16 \\
(0.33-2.33)\end{array}$ & $\begin{array}{c}1.41 \\
(0.83-4.38)\end{array}$ & $\begin{array}{c}1.65 \\
(0.97-3.17)\end{array}$ & $\begin{array}{c}1.84 \\
(1.14-3.52)\end{array}$ & $\begin{array}{c}1.97 \\
(1.46-3.56)\end{array}$ & $\begin{array}{c}2.14 \\
(1.48-3.35)\end{array}$ \\
\hline & SDR & $\begin{array}{c}4.43 \\
(2.07-5.57)^{*}\end{array}$ & $\begin{array}{c}3.54 \\
(2.57-5.08)^{*}\end{array}$ & $\begin{array}{c}3.17 \\
(2.23-5.54)\end{array}$ & $\begin{array}{c}3.19 \\
(1.79-4.68)\end{array}$ & $\begin{array}{c}2.24 \\
(1.95-3.70)\end{array}$ & $\begin{array}{c}2.81 \\
(2.35-4.03)\end{array}$ \\
\hline & VBF & $\begin{array}{c}11.51 \\
(4.14-16.52)^{*}\end{array}$ & $\begin{array}{c}7.08 \\
(4.25-13.98)^{*}\end{array}$ & $\begin{array}{c}8.83 \\
(5.65-13.21)^{*}\end{array}$ & $\begin{array}{c}5.83 \\
(3.98-10.79)^{*}\end{array}$ & $\begin{array}{c}4.63 \\
(2.55-6.10)^{*}\end{array}$ & $\begin{array}{c}3.61 \\
(2.75-5.61)^{*}\end{array}$ \\
\hline & FSXT & $\begin{array}{c}1.14 \\
(0.47-2.99)\end{array}$ & $\begin{array}{c}1.38 \\
(0.84-3.23)\end{array}$ & $\begin{array}{c}1.17 \\
(0.92-3.43)\end{array}$ & $\begin{array}{c}1.26 \\
(0.38-3.15)\end{array}$ & $\begin{array}{c}1.42 \\
(0.55-3.39)\end{array}$ & $\begin{array}{c}1.74 \\
(0.72-3.46)\end{array}$ \\
\hline & VDF & $\begin{array}{c}2.32 \\
(1.52-3.52)\end{array}$ & $\begin{array}{c}2.39 \\
(1.53-3.88)\end{array}$ & $\begin{array}{c}1.81 \\
(1.30-4.25)\end{array}$ & $\begin{array}{c}2.06 \\
(1.43-4.48)\end{array}$ & $\begin{array}{c}1.16 \\
(0.89-2.08)\end{array}$ & $\begin{array}{c}1.11 \\
(0.71-1.94)\end{array}$ \\
\hline & VF & $\begin{array}{c}1.34 \\
(0.56-2.55)\end{array}$ & $\begin{array}{c}1.73 \\
(0.93-2.88)\end{array}$ & $\begin{array}{c}2.10 \\
(0.51-2.92)\end{array}$ & $\begin{array}{c}2.07 \\
(0.65-3.06)\end{array}$ & $\begin{array}{c}2.06 \\
(0.65-3.34)\end{array}$ & $\begin{array}{c}2.56 \\
(0.63-3.56)\end{array}$ \\
\hline & FBF & $\begin{array}{c}2.14 \\
(1.23-3.32)\end{array}$ & $\begin{array}{c}2.13 \\
(1.53-3.29)\end{array}$ & $\begin{array}{c}2.19 \\
(1.38-3.39)\end{array}$ & $\begin{array}{c}2.21 \\
(1.66-2.94)\end{array}$ & $\begin{array}{c}2.82 \\
(2.13-3.46)\end{array}$ & $\begin{array}{c}2.83 \\
(2.08-4.03)\end{array}$ \\
\hline & TEF & $\begin{array}{c}0.64 \\
(0.45-1.41)\end{array}$ & $\begin{array}{c}0.85 \\
(0.64-1.69)\end{array}$ & $\begin{array}{c}1.10 \\
(0.91-2.44)\end{array}$ & $\begin{array}{c}1.24 \\
(0.90-2-16)\end{array}$ & $\begin{array}{c}1.95 \\
(1.39-4.02)\end{array}$ & $\begin{array}{c}2.27 \\
(1.93-2.61)\end{array}$ \\
\hline & SF & $\begin{array}{c}2.58 \\
(2.27-3.92) \\
\end{array}$ & $\begin{array}{c}2.66 \\
(2.19-3.38)\end{array}$ & $\begin{array}{c}2.75 \\
(2.18-3.74) \\
\end{array}$ & $\begin{array}{c}2.69 \\
(2.15-4.09) \\
\end{array}$ & $\begin{array}{c}2.71 \\
(2.43-3.30) \\
\end{array}$ & $\begin{array}{c}2.67 \\
(2.42-4.03)\end{array}$ \\
\hline
\end{tabular}

continue 


\begin{tabular}{|c|c|c|c|c|c|c|c|}
\hline \multirow{9}{*}{ Tea } & PF & $\begin{array}{c}7.96 \\
(7.02-9.86)^{*}\end{array}$ & $\begin{array}{c}11.79 \\
(10.10-13.67)^{*}\end{array}$ & $\begin{array}{c}14.06 \\
(12.51-16.62)^{*}\end{array}$ & $\begin{array}{c}15.85 \\
(13.79-17.74)^{*}\end{array}$ & $\begin{array}{c}19.16 \\
(17.44-21.20)^{*}\end{array}$ & $\begin{array}{c}20.97 \\
(18.90-23.18)^{*}\end{array}$ \\
\hline & SDR & $\begin{array}{c}12.89 \\
(11.88-14.54)^{*}\end{array}$ & $\begin{array}{c}15.07 \\
(13.47-17.07)^{*}\end{array}$ & $\begin{array}{c}17.37 \\
(15.5-20.13)^{*}\end{array}$ & $\begin{array}{c}18.32 \\
(16.69-20.49)^{*}\end{array}$ & $\begin{array}{c}21.54 \\
(19.98-24.18)^{*}\end{array}$ & $\begin{array}{c}23.77 \\
(21.36-25.90)^{*}\end{array}$ \\
\hline & VBF & $\begin{array}{c}21.99 \\
(16.75-31.80)^{*}\end{array}$ & $\begin{array}{c}28.23 \\
(24.40-35.02)^{*}\end{array}$ & $\begin{array}{c}32.55 \\
(28.85-37.15)^{*}\end{array}$ & $\begin{array}{c}32.52 \\
(30.56-36.11)^{*}\end{array}$ & $\begin{array}{c}38.94 \\
(36.45-40.69)^{*}\end{array}$ & $\begin{array}{c}41.03 \\
(38.49-42.58)^{*}\end{array}$ \\
\hline & FSXT & $\begin{array}{c}6.04 \\
(5.38-8.36)^{*}\end{array}$ & $\begin{array}{c}7.84 \\
(5.77-10.71)^{*}\end{array}$ & $\begin{array}{c}9.11 \\
(6.81-12.80)^{*}\end{array}$ & $\begin{array}{c}10.10 \\
(7.93-13.21)^{*}\end{array}$ & $\begin{array}{c}13.28 \\
(10.05-18.40)^{*}\end{array}$ & $\begin{array}{c}15.56 \\
(12.16-21.43)^{*}\end{array}$ \\
\hline & VDF & $\begin{array}{c}19.74 \\
(16.18-63.39)^{*}\end{array}$ & $\begin{array}{c}25.60 \\
(22.28-56.57)^{*}\end{array}$ & $\begin{array}{c}29.11 \\
(25.95-52.86)^{*}\end{array}$ & $\begin{array}{c}40.00 \\
(28.39-51.70)^{*}\end{array}$ & $\begin{array}{c}38.52 \\
(35.95-45.04)^{*}\end{array}$ & $\begin{array}{c}41.64 \\
(39.23-43.45)^{*}\end{array}$ \\
\hline & VF & $\begin{array}{c}3.72 \\
(2.81-7.00)^{*}\end{array}$ & $\begin{array}{c}4.01 \\
(2.89-7.22)^{*}\end{array}$ & $\begin{array}{c}4.68 \\
(3.95-7.54)^{*}\end{array}$ & $\begin{array}{c}7.97 \\
(6.90-10.93)^{*}\end{array}$ & $\begin{array}{c}14.52 \\
(12.45-16.66)^{*}\end{array}$ & $\begin{array}{c}21.64 \\
(19.73-24.81)^{*}\end{array}$ \\
\hline & FBF & $\begin{array}{c}16.36 \\
(14.18-18.26)^{*}\end{array}$ & $\begin{array}{c}22.57 \\
(19.67-25.10)^{*}\end{array}$ & $\begin{array}{c}28.30 \\
(24.79-31.30)^{*}\end{array}$ & $\begin{array}{c}31.00(27.91- \\
33.94)^{*}\end{array}$ & $\begin{array}{c}34.25 \\
(31.70-38.25)^{*}\end{array}$ & $\begin{array}{c}36.35 \\
(34.95-40.50)^{*}\end{array}$ \\
\hline & TEF & $\begin{array}{c}17.30 \\
(12.39-22.42)^{*}\end{array}$ & $\begin{array}{c}23.11 \\
(18.77-27.18)^{*}\end{array}$ & $\begin{array}{c}25.17 \\
(21.37-29.62)^{*}\end{array}$ & $\begin{array}{c}26.67 \\
(22.52-31.45)^{*}\end{array}$ & $\begin{array}{c}31.24(27.41- \\
36.41)^{*}\end{array}$ & $\begin{array}{c}31.74 \\
(28.31-36.66)^{*}\end{array}$ \\
\hline & SF & $\begin{array}{c}14.02 \\
(11.47-15.28)^{*}\end{array}$ & $\begin{array}{c}16.55 \\
(14.94-18.65)^{*}\end{array}$ & $\begin{array}{c}18.10 \\
(16.63-19.24)^{*}\end{array}$ & $\begin{array}{c}18.92 \\
(17.43-20.16)^{*}\end{array}$ & $\begin{array}{c}22.79 \\
(20.75-24.50)^{*}\end{array}$ & $\begin{array}{c}27.89 \\
(26.33-30.00)^{*}\end{array}$ \\
\hline \multirow{9}{*}{ Red wine } & PF & $\begin{array}{c}6.09 \\
(4.93-9.08)^{*}\end{array}$ & $\begin{array}{c}8.84 \\
(7.43-12.07)^{*}\end{array}$ & $\begin{array}{c}10.65 \\
(9.25-14.17)^{*}\end{array}$ & $\begin{array}{c}12.21 \\
(10.73-16.15)^{*}\end{array}$ & $\begin{array}{c}17.82 \\
(15.92-21.74)^{*}\end{array}$ & $\begin{array}{c}23.08 \\
(20.24-26.54)^{*}\end{array}$ \\
\hline & SDR & $\begin{array}{c}18.38 \\
(16.17-21.26)^{*}\end{array}$ & $\begin{array}{c}24.23 \\
(21.40-26.03)^{*}\end{array}$ & $\begin{array}{c}27.38 \\
(24.55-31.12)^{*}\end{array}$ & $\begin{array}{c}31.22 \\
(27.50-34.74)^{*}\end{array}$ & $\begin{array}{c}43.74 \\
(38.96-47.30)^{*}\end{array}$ & $\begin{array}{c}48.91 \\
(44.82-51.09)^{*}\end{array}$ \\
\hline & VBF & $\begin{array}{c}20.92 \\
(18.35-23.32)^{*}\end{array}$ & $\begin{array}{c}25.99 \\
(18.21-28.23)^{*}\end{array}$ & $\begin{array}{c}29.61 \\
(27.35-31.62)^{*}\end{array}$ & $\begin{array}{c}32.91 \\
(30.91-34.62)^{*}\end{array}$ & $\begin{array}{c}40.41 \\
(38.79-42.06)^{*}\end{array}$ & $\begin{array}{c}44.27 \\
(42.80-46.13)^{*}\end{array}$ \\
\hline & FSXT & $\begin{array}{c}6.35 \\
(5.23-8.25)^{*}\end{array}$ & $\begin{array}{c}7.29 \\
(5.87-10.15)^{*}\end{array}$ & $\begin{array}{c}8.28 \\
(7.04-10.55)^{*}\end{array}$ & $\begin{array}{c}8.86 \\
(7.39-12.38)^{*}\end{array}$ & $\begin{array}{c}12.81 \\
(10.69-15.11)^{*}\end{array}$ & $\begin{array}{c}16.48 \\
(14.31-19.59)^{*}\end{array}$ \\
\hline & VDF & $\begin{array}{c}20.39 \\
(17.67-23.02)^{*}\end{array}$ & $\begin{array}{c}26.83 \\
(24.09-29.15)^{*}\end{array}$ & $\begin{array}{c}31.13 \\
(28.31-33.25)^{*}\end{array}$ & $\begin{array}{c}34.49 \\
(31.42-37.50)^{*}\end{array}$ & $\begin{array}{c}43.07 \\
(40.09-45.12)^{*}\end{array}$ & $\begin{array}{c}45.11 \\
(43.76-47.07)^{*}\end{array}$ \\
\hline & VF & $\begin{array}{c}21.62 \\
(18.73-23.40)^{*}\end{array}$ & $\begin{array}{c}21.69 \\
(19.48-23.94)^{*}\end{array}$ & $\begin{array}{c}21.58 \\
(18.18-25.04)^{*}\end{array}$ & $\begin{array}{c}21.73 \\
(19.66-23.12)^{*}\end{array}$ & $\begin{array}{c}19.91 \\
(16.88-51.36)^{*}\end{array}$ & $\begin{array}{c}24.09 \\
(20.52-27.54)^{*}\end{array}$ \\
\hline & $\mathrm{FBF}$ & $\begin{array}{c}21.01 \\
(17.41-24.70)^{*}\end{array}$ & $\begin{array}{c}24.23 \\
(20.74-27.09)^{*}\end{array}$ & $\begin{array}{c}29.56 \\
(24.11-31.64)\end{array}$ & $\begin{array}{c}30.01 \\
(26.19-33.48)^{*}\end{array}$ & $\begin{array}{c}39.17 \\
(36.25-41.87)^{*}\end{array}$ & $\begin{array}{c}43.08 \\
(40.73-44.41)^{*}\end{array}$ \\
\hline & TEF & $\begin{array}{c}20.77 \\
(19.00-22.72)^{*}\end{array}$ & $\begin{array}{c}26.01 \\
(17.94-28.42)^{*}\end{array}$ & $\begin{array}{c}28.28 \\
(26.00-29.78)^{*}\end{array}$ & $\begin{array}{c}30.11 \\
(28.09-31.87)^{*}\end{array}$ & $\begin{array}{c}34.72 \\
(33.29-36.10)^{*}\end{array}$ & $\begin{array}{c}37.90 \\
(35.81-39.06)^{*}\end{array}$ \\
\hline & SF & $\begin{array}{c}9.53 \\
(8.91-11.59)^{*}\end{array}$ & $\begin{array}{c}11.79 \\
(11.24-14.07)^{*}\end{array}$ & $\begin{array}{c}14.25 \\
(12.85-17.17)^{*}\end{array}$ & $\begin{array}{c}14.82 \\
(13.82-17.43)^{*}\end{array}$ & $\begin{array}{c}25.29 \\
(22.72-30.71)^{*}\end{array}$ & $\begin{array}{c}29.45 \\
(26.51-33.39)^{*}\end{array}$ \\
\hline \multirow{9}{*}{ Orange Juice } & PF & $\begin{array}{c}2.78 \\
(1.93-3.37)\end{array}$ & $\begin{array}{c}3.79 \\
(3.44-5.71)^{*}\end{array}$ & $\begin{array}{c}4.67 \\
(4.35-5.86)^{*}\end{array}$ & $\begin{array}{c}5.40 \\
(4.87-6.08)^{*}\end{array}$ & $\begin{array}{c}9.39 \\
(8.35-10.93)^{*}\end{array}$ & $\begin{array}{c}14.39 \\
(12.68-16.27)^{*}\end{array}$ \\
\hline & SDR & $\begin{array}{c}4.65 \\
(2.98-5.46)^{*}\end{array}$ & $\begin{array}{c}6.83 \\
(5.67-8.11)^{*}\end{array}$ & $\begin{array}{c}7.14 \\
(5.06-8.18)^{*}\end{array}$ & $\begin{array}{c}7.87 \\
(6.39-9.19)^{*}\end{array}$ & $\begin{array}{c}11.13 \\
(10.06-12.78)^{*}\end{array}$ & $\begin{array}{c}15.75 \\
(13.96-17.38)^{*}\end{array}$ \\
\hline & VBF & $\begin{array}{c}5.66 \\
(3.82-7.33)^{*}\end{array}$ & $\begin{array}{c}4.58 \\
(3.80-9.37)^{*}\end{array}$ & $\begin{array}{c}5.45 \\
(4.56-7.16)^{*}\end{array}$ & $\begin{array}{c}6.47 \\
(5.52-8.85)^{*}\end{array}$ & $\begin{array}{c}11.56 \\
(10.26-12.43)^{*}\end{array}$ & $\begin{array}{c}19.30 \\
(17.83-20.56)^{*}\end{array}$ \\
\hline & FSXT & $\begin{array}{c}2.84 \\
(2.22-4.10)\end{array}$ & $\begin{array}{c}4.03 \\
(3.31-5.51)^{*}\end{array}$ & $\begin{array}{c}5.06 \\
(3.32-6.95)^{*}\end{array}$ & $\begin{array}{c}5.77 \\
(4.94-7.53)^{*}\end{array}$ & $\begin{array}{c}9.22 \\
(7.69-11.46)^{*}\end{array}$ & $\begin{array}{c}12.39 \\
(10.04-15.31)^{*}\end{array}$ \\
\hline & VDF & $\begin{array}{c}2.61 \\
(1.18-3.82)\end{array}$ & $\begin{array}{c}4.32 \\
(2.32-5.44)^{*}\end{array}$ & $\begin{array}{c}5.74 \\
(4.34-6.44)^{*}\end{array}$ & $\begin{array}{c}6.72 \\
(4.49-8.54)^{*}\end{array}$ & $\begin{array}{c}10.41 \\
(8.88-12.11)^{*}\end{array}$ & $\begin{array}{c}16.34 \\
(15.10-19.42)^{*}\end{array}$ \\
\hline & VF & $\begin{array}{c}1.77 \\
(0.87-2.55)\end{array}$ & $\begin{array}{c}2.37 \\
(1.93-3.20)\end{array}$ & $\begin{array}{c}2.75 \\
(2.19-3.47)\end{array}$ & $\begin{array}{c}3.42 \\
(2.49-4.14)^{*}\end{array}$ & $\begin{array}{c}5.03 \\
(4.49-6.57)^{*}\end{array}$ & $\begin{array}{c}8.32 \\
(7.62-9.92)^{*}\end{array}$ \\
\hline & FBF & $\begin{array}{c}4.33 \\
(3.89-5.76)^{*}\end{array}$ & $\begin{array}{c}8.70 \\
(7.42-9.87)^{*}\end{array}$ & $\begin{array}{c}10.51 \\
(9.57-11.40)^{*}\end{array}$ & $\begin{array}{c}11.58 \\
(10.66-12.83)^{*}\end{array}$ & $\begin{array}{c}18.09 \\
(17.16-19.81)^{*}\end{array}$ & $\begin{array}{c}20.93 \\
(19.83-22.67)^{*}\end{array}$ \\
\hline & TEF & $\begin{array}{c}4.19 \\
(3.27-4.86)^{*}\end{array}$ & $\begin{array}{c}6.07 \\
(4.84-6.65)^{*}\end{array}$ & $\begin{array}{c}6.73 \\
(5.80-7.35)^{*}\end{array}$ & $\begin{array}{c}7.64 \\
(6.73-8.44)^{*}\end{array}$ & $\begin{array}{c}13.77 \\
(11.99-15.57)^{*}\end{array}$ & $\begin{array}{c}17.37 \\
(15.31-19.17)^{*}\end{array}$ \\
\hline & SF & $\begin{array}{c}5.26 \\
(4.13-5.85)^{*}\end{array}$ & $\begin{array}{c}6.36 \\
(5.50-7.07)^{*}\end{array}$ & $\begin{array}{c}7.38 \\
(6.76-8.29)^{*}\end{array}$ & $\begin{array}{c}8.47 \\
(7.42-9.48)^{*}\end{array}$ & $\begin{array}{c}18.38 \\
(16.35-22.17)^{*}\end{array}$ & $\begin{array}{c}22.62 \\
(21.34-24.35)^{*}\end{array}$ \\
\hline
\end{tabular}

PF: Premise Flowable; SDR: Smart Dentin Replacement; VBF: Venus Bulk Fill; FSXT: Filtek Supreme XTE; VDF: Venus Diamond Flow; VF: Vertise Flow; FBF: Filtek Bulk Fill; TEF: Tetric EvoFlow; SF: Sonic Fill; T1: 1 week; T2: 2 weeks; T3: 3 weeks; T4: 4 weeks; T5: 3 months; T6: 6 months. *Indicates clinically unacceptable values $(\triangle \mathrm{E}>3.3)$. 

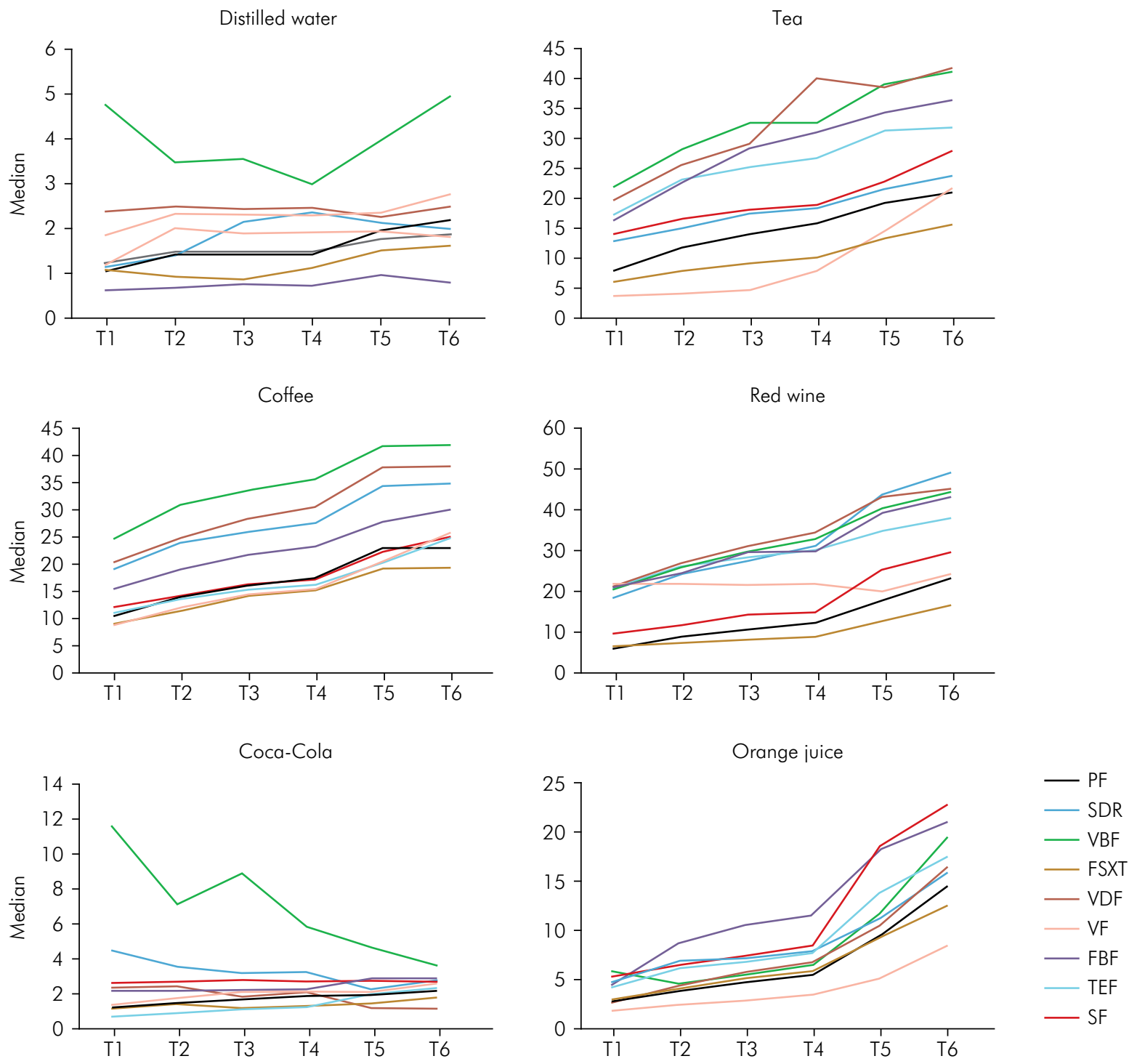

Figure. Color change progression of each composite and staining solution at the different time points.

According to composite type, the bulk-fill group $\left(\mathrm{W}_{\mathrm{S}}=18.84 \pm 5.20 ; \mathrm{W}_{\mathrm{L}}=2.13 \pm 2.93\right)$ exhibited the lowest water sorption and solubility values, the nanohybrid group exhibited intermediate values $\left(\mathrm{W}_{\mathrm{S}}=24.52 \pm 5.17 ; \mathrm{W}_{\mathrm{L}}=2.31 \pm 2.99\right)$, and the self-adhesive group exhibited the highest values $\left(\mathrm{W}_{\mathrm{S}}=69.10 \pm 7.19 ; \mathrm{W}_{\mathrm{L}}=11.20 \pm 10.76\right)$.

The Pearson analysis showed a positive statistically significant correlation between water sorption and solubility $(\mathrm{r}=0.569, \mathrm{p}<0.005)$. A positive correlation was also observed between water sorption and $\Delta \mathrm{E}(\mathrm{r}=0.059$, $\mathrm{p}=0.338)$ and between solubility and $\Delta \mathrm{E}(\mathrm{r}=0.039$, $\mathrm{p}=0.528)$ but these values were not statistically significant.

\section{Discussion}

Composite resin materials are inevitably exposed to saliva, food and beverages in the oral environment; these factors affect color change as well as oral hygiene $e^{3,27,28}$ and the surface smoothness of the restoration. ${ }^{3,21,28}$ Natural saliva has a protective effect because it forms a surface barrier that limits staining ${ }^{27}$ and dilutes staining solutions. ${ }^{23}$ Because there is no effective way to simulate the mouth with fresh saliva, the present study used distilled water, although saliva would be expected to present a much better protective effect. 
Table 3. Results of water sorption and solubility.

\begin{tabular}{lccc}
\hline $\begin{array}{l}\text { Composite } \\
\text { type }\end{array}$ & Composite & $\begin{array}{c}\text { Water sorption } \\
(\mu \mathrm{g} / \mathrm{mm} 3)\end{array}$ & $\begin{array}{c}\text { Water solubility } \\
(\mu \mathrm{g} / \mathrm{mm} 3)\end{array}$ \\
\hline \multirow{3}{*}{ Bulk fill } & FBF & $22.20(3.06)^{\mathrm{c}}$ & $0.65(2.41)^{\mathrm{ab}}$ \\
& SDR & $19.21(4.10)^{\mathrm{b}}$ & $2.72(1.76)^{\mathrm{d}}$ \\
& VBF & $21.41(3.89)^{\mathrm{c}}$ & $0.22(2.69)^{\mathrm{a}}$ \\
& $\mathrm{SF}$ & $12.53(3.16)^{\mathrm{a}}$ & $4.94(2.12)^{\mathrm{e}}$ \\
& FSXT & $22.49(3.30)^{\mathrm{c}}$ & $2.60(3.12)^{\mathrm{cd}}$ \\
Nanohybrid & TEF & $29.59(3.86)^{\mathrm{e}}$ & $3.96(2.81)^{\mathrm{e}}$ \\
& $\mathrm{PF}$ & $24.42(5.61)^{\mathrm{d}}$ & $1.16(3.23)^{\mathrm{ab}}$ \\
& VDF & $21.57(3.53)^{\mathrm{c}}$ & $1.51(1.89)^{\mathrm{bc}}$ \\
Self-adhesive & VF & $69.10(7.19)^{f *}$ & $11.20(10.76)^{\mathrm{f**}}$ \\
\hline
\end{tabular}

FBF: Filtek Bulk Fill; SDR: Smart Dentin Replacement; VBF: Venus Bulk Fill; SF: Sonic Fill; FSXT: Filtek Supreme XTE; TEF: Tetric EvoFlow; PF: Premise Flowable; VDF: Venus Diamond Flow; VF: Vertise Flow. Same superscript letters in the same column indicate no statistically significant differences. ${ }^{*}$ Indicates values of water sorption $>40 \mu \mathrm{g} / \mathrm{mm}^{3}$

${ }^{* *}$ Indicates values of water solubility $>7.5 \mu \mathrm{g} / \mathrm{mm}^{3}$

Finishing/polishing procedures may also affect the composite surface quality; therefore, they are linked to the early discoloration of resin composites. ${ }^{18}$ To standardize and achieve the smoothest surface possible, a polyester strip was used to create a surface rich in resin, which is representative of the clinical situation when matrices are used. ${ }^{3,21,24}$ The effect of finishing/polishing techniques on discoloration should be considered in future long-term in vitro studies.

Red wine, coffee, tea, orange juice and Coca-Cola are common beverages in the modern diet, and some have a staining potential for restorative materials. ${ }^{3,23,24}$ The results of the present study demonstrated this staining potential, as in other studies. ${ }^{1,2,3,4,18,24,27,28}$

Extrinsic discoloration can be removed by daily brushing, a good finishing/polishing technique, and bleaching agents. Some discoloration is easier to remove than others. ${ }^{2,3,18,20,28}$ Although extrinsic factors cause the most discoloration, intrinsic factors involved in the staining process must be considered because they are irreversible and cannot be removed nor bleached, although teeth can be successfully bleached.

The perceived color match of the material to the tooth might be acceptable even though the material is changing color because the tooth could be changing color in a similar pattern with aging. The superior color matching of VF (48 vol\%), SF (66.8 vol\%), PF
(70 vol\%) and FSXT (46 vol\%) may be related to their filler content. These composites contain $\geq 70 \%$ wt filler; some studies ${ }^{2,22}$ have shown that composites with a high filler content exhibit superior color matching. Ytterbium trifluoride, particles that contribute to fluoride release, is a water-soluble component that leaches from composites after immersion in a solution. ${ }^{2,22}$ This may have affected the discoloration of TEF, FBF and VDF in this study.

VDF had worse color matching than TEF, PF and FSXT but exhibited similar color change to some bulk-fill materials in the universal shade and to VDF in the A2 shade. This color change may be attributable to the silane agent coupling the filler particles and resin matrix because VDF and some bulk-fill composites (SDR and VBF) contain a mixture of urethane dimethacrylate (UDMA) and ethoxylated bisphenol A dimethacrylate (EBPADMA) in their matrix. These two monomers are described in the literature as hydrophobic ${ }^{2,3,29}$ and UDMA reportedly exhibits a low $\Delta \mathrm{E}^{2,19}$ Some studies ${ }^{19,23}$ have suggested that the silanization of filler particles contributes to discoloration as a result of silane's high propensity for water sorption. This could be related to the varied and complex reactions of silane. The shade of the composite is an additional factor because darker shades exhibit better color matching due to the presence of pigments. Conceivably, universal shades undergo a higher degree of color change $\mathrm{e}^{15,16}$ because of the absence of pigments. In addition, Uchida et al. ${ }^{16}$ reported that the greater discoloration associated with lighter shades might result from an environmental breakdown of the polymer, leading to the release of monomers and color change, or from an environmental effect on the retention of pigments and other additives.

In general, the composites included in this study had a $\Delta \mathrm{E} \leq 3.3$, considered clinically acceptable, in distilled water and Coca-Cola. . $^{1,218,19,24}$ Only VBF showed a $\Delta \mathrm{E} \geq 3.3$ in these solutions. These values could be related to their universal shade, lower levels of filler content or the degradation of silane. In contrast, orange juice, coffee, tea and red wine resulted in a $\Delta \mathrm{E} \geq 3.3$ in all composites studied. ${ }^{3,18,23,27}$

According to the estimation of Ertaş et al., ${ }^{18}$ the 6-month immersion period chosen for this study is 
equivalent to approximately 13 years of clinical ageing (24 h of staining in vitro corresponds to approximately 1 month in vivo). Thus, if 17-22 years is considered the expected lifespan of modern composite resin materials, ${ }^{30}$ the immersion period used in this study is clinically relevant.

Water sorption and solubility are linked to the composition of resin matrices and, in addition to exerting deleterious effects on their structure and function, may represent precursors to a variety of chemical and physical processes of biological concern. ${ }^{25}$ Since the first materials were introduced, mixtures of dimethacrylate monomers such as Bisphenol A diglycidyl ether dimethacrylate (Bis-GMA), Bisphenol A polyethylene glycol diether dimethacrylate (Bis-EMA) and UDMA have been commonly incorporated into most commercial resin composites. However, in recent years, new organic matrix monomers have been introduced, such as silorane, DX-511 monomer, ${ }^{11}$ and EBPADMA, ${ }^{29}$ as well as mixtures of self-adhesive monomers in methacrylate such as glycerol phosphate dimethacrylate (GPDM) $)^{10,11}$ and pyromellitic glycerol dimethacrylate (PMGDM). ${ }^{29}$

VF, which contains GPDM, a group of adhesive monomers that was commercially introduced by Buonacuore, ${ }^{11}$ showed the highest water sorption. The structure of the adhesive monomers influences the hydrophilicity of the resulting polymer. ${ }^{11}$ Therefore, the hydrophilic acidic phosphate group and the structure of GPDM may underlie the degree of water sorption by this composite. ${ }^{10,11}$ Other factors associated with water sorption are the type and quantity (\% vol or $\% \mathrm{wt}$ ) of filler particles used. Wei et al. ${ }^{10,11}$ reported that, as the only flowable composite examined in their study, VF contained less filler and more monomer than other materials tested. All the composites tested in this study were flowable and contained more or less the same amount of filler. However, although VF contains the same type of particles as PF, their degree of water sorption differed.

Some authors maintain that color change is associated with the water sorption and hydrophilicity of the resin matrix. ${ }^{21,22,23}$ However, our study showed that VF had the highest water sorption whereas the color change was similar to the SF and nanohybrid groups, excluding VDF. Although the Pearson's correlation was positive, it showed no statistically significant differences between water sorption and color change. These findings could be less related to the resin matrix composition than the filler content and pigments in the formulation.

The bulk-fill and nanohybrid composites showed similar levels of water sorption in our study. In general, the matrix composition and filler content were comparable between these groups, which may explain the similarity between these values. ${ }^{31}$ However, the difference in $\Delta \mathrm{E}$ values could be related to the absence of pigments and greater translucency in bulk-fill composites.

$\mathrm{SF}$, which exhibited the lowest water sorption of all the materials, is a new bulk-fill composite that requires sonic activation. The sonic energy, applied through a special handpiece to increase flowability, ${ }^{13}$ may have reduced the water sorption of SF, which also had the highest filler content of the materials tested. These results may be related to filler content and components of the resin matrix such as Bis-EMA.

With the exception of VF, the water sorption levels of the composites tested in this study were within the parameters stipulated by ISO4049:200926 and consistent with published studies. Furthermore, the color match results determined in this study concurred with the literature regarding the tendency of composites to undergo color changes in different beverages. However, a high degree of water sorption did not equate with a significant color change; different factors influenced color alteration, such as the pigments used, filler content or temperature.

Some authors consider that high temperatures $\left(50^{\circ} \mathrm{C}\right.$ and $\left.70^{\circ} \mathrm{C}\right)$, which may promote further polymerization ${ }^{17}$, cause surface degradation ${ }^{20}$ and affect color change. ${ }^{6}$ The influence of the temperature of beverages on color change represents a topic for future research.

\section{Conclusion}

Within the limitations of this in vitro study, the null hypotheses were accepted. Significant differences in color change were evident when the materials were compared by composite type. The bulk-fill type showed the highest discoloration $(\Delta \mathrm{E}=14.21$ 
(0.17-51.09) and the nanohybrid type showed the lowest $(\Delta \mathrm{E}=8.55(0.22-63.39))$. The self-adhesive type absorbed more water $\left(\mathrm{W}_{\mathrm{S}}=69.10 \pm 7.19\right)$. The findings of the present study suggest that water sorption is one factor associated with the ability of composites to discolor, though discoloration is a multifactorial problem.

\section{References}

1. Catelan A, Briso AL, Sundfeld RH, Goiato MC, Santos PH. Color stability of sealed composite resin restorative materials after ultraviolet artificial aging and immersion in staining solutions. J Prosthet Dent. 2011;105(4):236-41. doi:10.1016/S0022-3913(11)60038-3

2. Nasim I, Neelakantan P, Sujeer R, Subbarao CV. Color stability of microfilled, microhybrid and nanocomposite resins: -an in vitro study. J Dent. 2010;38 Suppl 2: e137-42. doi:10.1016/j.jdent.2010.05.020

3. Ardu S, Braut V, Gutemberg D, Krejci I, Dietschi D, Feilzer AJ. A long-term laboratory test on staining susceptibility of esthetic composite resin materials. Quintessence Int. 2010;41(8):695-702. doi:10.1016/j.jdent.2011.12.017

4. Barutcigil Ç, Yildiz E. Intrinsic and extrinsic discoloration of dimethacrylate and silorane based composites. J Dent. 2012;40 Suppl 1:e57-63. doi:10.1016/j.jdent.2011.12.017

5. Zimmerli B, Strub M, Jeger F, Stadler O, Lussi A. Composite materials: composition, properties and clinical applications: a literature review. Schweiz Monatsschr Zahnmed. 2010;120(10):972-86.

6. Arregui M, Giner L, Ferrari M, Mercadé M. Colour stability of self-adhesive flowable composites before and after storage in water. Key Eng Mater. 2015;631(1):143-50.

7. Beun S, Bailly C, Devaux J, Leloup G. Rheological properties of flowable resin composites and pit and fissure sealants. Dent Mater. 2008;24(4):548-55. doi:10.1016/j.dental.2007.05.019

8. Cadenaro M, Marchesi G, Antoniolli F, Davidson C, De Stefano Dorigo E, Breschi L. Flowability of composites is no guarantee for contraction stress reduction. Dent Mater. 2009;25(5):649-54. doi:10.1016/j.dental.2008.11.010

9. Salerno M, Derchi G, Thorat S, Ceseracciu L, Ruffilli $\mathrm{R}$, Barone AC. Surface morphology and mechanical properties of new-generation flowable resin composites for dental restoration. Dent Mater. 2011;27(12):1221-8. doi:10.1016/j.dental.2011.08.596

10. Wei YJ, Silikas N, Zhang ZT, Watts DC. Hygroscopic dimensional changes of self-adhering and new resin-matrix composites during water sorption/desorption cycles. Dent Mater. 2011;;27(3):259-66. doi:10.1016/j.dental.2010.10.015

\section{Acknowledgments}

The authors would like to thank the following manufacturers for providing the materials tested in this study: Kerr Corp, Ivoclar Vivadent, Dentsply, and 3M ESPE. The authors would also like to thank Mark Lodge for his help in English revision and Dr. Jaume Llopis for his support in statistical analysis.

11. Wei YJ, Silikas N, Zhang ZT, Watts DC. Diffusion and concurrent solubility of self-adhering and new resin-matrix composites during water sorption/desorption cycles. Dent Mater. 2011;27(2):197-205. doi:10.1016/j.dental.2010.10.014

12. Vichi A, Margvelashvili M, Goracci C, Papacchini F, Ferrari M. Bonding and sealing ability of a new self-adhering flowable composite resin in class I restorations. Clin Oral Investig. 2013;17(6):1497-506. doi:10.1007/s00784-012-0846-6

13. Conte NR Jr, Goodchild JH. Flowable composite resins: do they decrease microleakage and shrinkage stress? Compend Contin Educ Dent. 2013;34(Spec No 4):1-6.

14. Yu B, Lee YK. Differences in color, translucency and fluorescence between flowable and universal resin composites. J Dent. 2008;36(10):840-6. doi:10.1016/j.jdent.2008.06.003

15. Korkmaz Ceyhan Y, Ontiveros JC, Powers JM, Paravina RD. Accelerated aging effects on color and translucency of flowable composites. J Esthet Restor Dent 2014;26(4):272-8. doi:10.1111/jerd.12093

16. Uchida H, Vaidyanathan J, Viswanadhan $T$, Vaidyanathan TK. Color stability of dental composites as a function of shade. J Prosthet Dent. 1998;79(4):372-7. doi:10.1016/S0022-3913(98)70147-7

17. Nakamura T, Saito O, Mizuno M, Tanaka H. Changes in translucency and color of particulate filler composite resins. Int J Prosthodont. 2002;15(5):494-9.

18. Ertaş E, Güler AU, Yücel AÇ, Köprülü H, Güler E. Color stability of resin composites after immersion in different drinks. Dent Mater J. 2006;25(2):371-6. doi:10.4012/dmj.25.371

19. Mundim FM, Garcia LF, Pires-de-Souza FC. Effect of staining solutions and repolishing on color stability of direct composites. J Appl Oral Sci. 2010;18(3):249-54. doi:10.1590/S1678-77572010000300009

20. Tuncer D, Karaman E, Firat E. Does the temperature of beverages affect the surface roughness, hardness, and color stability of a composite resin? Eur J Dent. 2013;7(2):165-71. doi:10.4103/1305-7456.110161 
21. Ergücü Z, Türkün LS, Aladag A. Color stability of nanocomposites polished with one-step systems. Oper Dent. 2008;33(4):413-20. doi:10.2341/07-107

22. Dietschi D, Campanile G, Holz J, Meyer JM. Comparison of the color stability of ten new-generation composites: an in vitro study. Dent Mater. 1994;10(6):353-62. doi:10.1016/0109-5641(94)90059-0

23. Bagheri R, Burrow MF, Tyas M. Influence of food-simulating solutions and surface finish on susceptibility to staining of aesthetic restorative materials. J Dent. 2005;33(5):389-98. doi:10.1016/j.jdent.2004.10.018

24. Arocha MA, Mayoral JR, Lefever D, Mercadé M, Basilio J, Roig M. Color stability of siloranes versus methacrylate-based composites after immersion in staining solutions. Clin Oral Investig. 2013;17(6):1481-7. doi:10.1007/s00784-012-0837-7

25. Ferracane JL. Hygroscopic and hydrolytic effects in dental polymer networks. Dent Mater. 2006;22(3):211-22. doi:10.1016/j.dental.2005.05.005
26. International Organization for Standardization. ISO 4049-2009: Dentistry: polymer-based restorative materials. Geneva: International Organization for Standardization; 2009.

27. Omata Y, Uno S, Nakaoki Y, Tanaka T, Sano H, Yoshida S et al. Staining of hybrid composites with coffee, oolong tea, or red wine. Dent Mater J. 2006;25(1):125-31. doi:10.4012/dmj.25.125

28. Fujita M, Kawakami S, Noda M, Sano H. Color change of newly developed esthetic restorative material immersed in food-simulating solutions. Dent Mater J. 2006;25(2):352-9. doi:10.4012/dmj.25.352

29. Dickens SH, Flaim GM, Floyd CJ. Effects of adhesive, base and diluent monomers on water sorption and conversion of experimental resins. Dent Mater. 2010;26(7):675-81. doi:10.1016/j.dental.2010.03.011

30. Ferracane JL. Resin-based composite performance: are there some things we can't predict? Dent Mater. 2013;29(1):51-8. doi:10.1016/j.dental.2012.06.013

31. Alrahlah A, Silikas N, Watts DC. Hygroscopic expansion kinetics of dental resin-composites. Dent Mater. 2014;30(2):143-8. doi:10.1016/j.dental.2013.10.010 